\title{
3D Functional Database of Subcortical Structures for Surgical Guidance in Image Guided Stereotactic Neurosurgery
}

\author{
K.W. Finnis ${ }^{1}$, Y.P. Starreveld ${ }^{1,2}$, A.G. Parrent ${ }^{2}$, A.F. Sadikot ${ }^{3}$, and \\ T.M. Peters ${ }^{1}$ \\ 1 John P. Robarts Research Institute, University of Western Ontario \\ Box 5015, 100 Perth Drive, London ON N6A 5K8, Canada \\ ${ }^{2}$ London Health Sciences Center, London, ON Canada \\ 3 Montreal Neurological Institute, Montréal, QC, Canada \\ kfinnis@irus.rri.on.ca
}

\begin{abstract}
Current techniques for deep brain stereotactic neurosurgery require identification of targets by preoperative imaging localization. Many critical structures targeted in this way (the thalamic nuclei) are functionally distinct but not discernable on magnetic resonance images. These structures are also surrounded by critical brain areas which must not be damaged by the surgical procedure. These factors make accurate localization of lesion targets crucial. Digitized anatomical atlases derived from histochemically stained brain specimens registered to patient MRI datasets aid in delineating targets but accuracy of registration withinhomogeneous anatomy remains questionable. To address this problem, we have designed a searchable and expandable database of functional organization for the sensorimotor thalamus, internal capsule, and internal pallidum from a population of patients $(n=40)$. Data were obtained through microcellular recording, microstimulation, and macrostimulation mapping performed during stereotactic thalamotomies and pallidotomies. After registration of the database into standard stereotactic space, clustering of like physiological responses was noted in the internal capsule and sensorimotor thalamus and an articulated joint-based organization was observed in the internal pallidum. Furthermore, a clear delineation of the kinesthetic-paresthetic functional border was observed within the thalamus. When registered to a patient MRI within our image guided visualization platform, the database provides a visual representation of deep brain functional organization facilitating physiological exploration and preoperative planning.
\end{abstract}

\section{Introduction}

\subsection{Surgical Procedure and Target Localization}

The main surgical procedure for Parkinson's Disease (PD) involves stereotactic localization and lesioning of discreet areas within the midbrain. For over 50 years

C. Taylor, A. Colchester (Eds.): MICCAI'99, LNCS 1679, pp. 758-767, 1999.

(C) Springer-Verlag Berlin Heidelberg 1999 
now, stereotactic thalamotomy for relief of contralateral tremor has been the primary surgical treatment for parkinsonian tremor $[1,2]$. In this procedure, the Ventralis intermedius (Vim) nucleus of the thalamus is lesioned via leukotome lesioning or radiofrequency thermocoagulation. Lesioning of this motor nucleus eliminates the excessive and abnormal activity associated with it in the parkinsonian state and as a result, tremor is alleviated. Pallidotomy, or lesioning of the globus pallidus internus(GPi), was first performed 40 years ago with a resurgence in interest over the last 10 years. Lesioning the GPi has proven to greatly reduce contralateral limb rigidity and the accompanying dyskinesias associated with PD $[1,2,3]$. Currently, the posteroventral aspect of the GPi and the Vim nucleus of the thalamus constitute the desired targets for pallidotomy and thalamotomy respectively at both the Montreal Neurological Institute (MNI) and the London Health Sciences Center (LHSC).

Target localization is based upon preoperative magnetic resonance imaging. A stereotactic head frame equipped with fiducial markers filled with copper sulfate solution is rigidly affixed to the patient's skull prior to scanning. The fiducial markers are visible on the resulting MRI images and provide the neurosurgeon with a reference for calculation of a frame-to-MRI coordinate transformation. An arc system mounted on top of the frame provides a means of attaching and orienting surgical tools during the procedure while a small hole drilled through the skull provides access to the calculated target structure coordinates. Since this is an arc-centered system, once the depth of the surgical tool is locked into position, trajectory angles may change while the tip of the tool remains on target.

The benefits of electrophysiological confirmation for radiographical localization have been demonstrated in the past by several investigators [1,4,5]. Electrophysiological exploration helps localize the target by locating characteristic firing patterns. Surgeons may also appreciate and adjust for the inherent distortion found in the patient MRI due to field inhomogeneities using the results of exploration. As described below, three different electrophysiological methods are employed during this procedure: microcellular recording (LHSC), microstimulation (LHSC), and macrostimulation (LHSC, MNI). Each method is used to locate and map the specific somatotopic organization within deep brain anatomy.

\subsection{Microcellular Recording}

Mapping deep brain structures with microcellular recording electrodes identifies neurons that produce characteristic firing patterns in response to specific stimuli. Kinesthetic neurons are cells which respond to movement around articulated joints and are detected by sharp excitation or inhibition of firing rates in the presence of joint movement. Only those joints which exist within the somatotopic domain of that kinesthetic neuron evoke cell firing [5,6]. Tactile neurons are cells which are activated by light touch to skin within their receptive field. When the receptive field of a tactile neuron is touched, rapid cell firing of that neuron is produced. Typically, a neurosurgeon introduces a recording electrode into the thalamus or GPi to record neuronal firing patterns both deep and superficial to the target coordinate along each trajectory. In the case of a thalamotomy, 
kinesthetic neurons are detected throughout the Vim until the functional border between the Vim and the Ventral caudal (Vc) sensory nucleus, is traversed. When kinesthetic neurons are encountered along a trajectory, electrode depth and the stimuli which evoked the neuronal response are catalogued by the surgeon in the surgical log. Since thalamic nuclei are somatotopically organized [4,6], every data point collected aids the surgeon in mentally reconstructing the somatotopic organization contained within that nucleus, and in establishing the functional borders which separate them. During a pallidotomy, microcellular recording is performed in much the same way although pure sensory neurons are not present in this area of the brain. The somatotopic organization of the GPi has previously been described, based on a small patient sample, as kinesthetic joint-based with only partial somatotopy observable [7].

A distinct benefit of using microcellular recording is its ability to precisely locate tremor cells [8]. Tremor cells, a term coined by Guiot et al. in the early sixties, refer to neurons which fire in synchrony with patient tremor, typically in the range of 3 to $5 \mathrm{~Hz}$ in PD. Subsequent macrostimulation within regions containing tremor cells usually abates or arrests all signs of physiologic tremor contralaterally.

\subsection{Microstimulation}

By introducing a small electrical current within a discreet area of brain, neuronal membranes will depolarize beyond threshold resulting in cell excitation. When microstimulation supplies enough current to reach neuronal threshold and excite a small pool of neurons, the effects of stimulation are immediately demonstrated by the fully aware patient, usually in the form of paresthesias or muscle contractions. During thalamotomies, it has been demonstrated that microstimulation within the Vim do not induce muscle contractions, although on rare occasions, a sensation of movement within stationary limbs may be induced [4]. Responses are reported by patients immediately after the sensory Vc nucleus of the thalamus is stimulated at suprathreshold levels. The $\mathrm{Vc}$ receives afferents primarily from the spinothalamic tract relaying information pertaining to temperature, vibration, superficial sensation and pain pathways. Typical patient descriptions after stimulation within the Vc nucleus include tingling, electrical, or pins and needles and may range from a localized pleasant numbness to occasional sharp pain or burning sensations. Direct control over stimulation current by the surgeon enables determination of neuronal thresholds and control of the extent of stimulation influence within the brain tissue. All verbal responses provided by the patient are entered into the surgical log with microelectrode depth and amount of current which evoked the response. Microstimulation confirms the functional border between the Vim nucleus (no response) and its caudal neighbor the Vc nucleus (induced paresthesia) in the sagittal plane parallel to the probe trajectory. Microstimulation is incorporated into the pallidotomy procedure to test for proximity of the optic tract and in establishing the border between the internal capsule and the GPi. Patients experience visual phenomena such as homony- 
mous scotoma or phosphenes during optic tract stimulation and demonstrate contractions of muscles during capsular stimulation.

\subsection{Macrostimulation}

Macrostimulation introduces higher voltages into the brain through the noninsulated tip of the stimulation electrode. Macrostimulation commonly ranges in frequency from 60 to $200 \mathrm{H}$ with the surgeon in control of the voltage introduced into the brain (usually between 0.25 and $4 \mathrm{~V}$ ). Application of increasing voltage includes more neurons in the activated pool and obvious patient responses may be elicited. Macrostimulation is the method of choice at the MNI for mapping both motor responses obtained in the internal capsule for use as physiological landmarks and for evoking paresthetic responses within the Vc. Macrostimulation is also quite effective in demonstrating reversible lesions within an area of the Vim. When high frequency stimulation is applied to a pool of tremor cells, for example, these neurons cease firing and tremor arrest occurs within the body parts localized in that somatotopic area [9]. Once stimulation subsides, physiological function returns to prior levels.

\subsection{Anatomical Atlases}

In order to facilitate identification of invisible anatomical targets, stereotactic anatomical maps created from stained sections of postmortem brains are often consulted. The Schaltenbrand Wahren atlas [10] is based on stained postmortem brain slices which clearly display thalamic nuclear borders and surrounding structures. This atlas presents slices in sagittal, coronal, and axial planes and is frequently used in preoperative planning. Several groups, including the MNI, have digitized the Schaltenbrand Wahren atlas for superposition over patient MRI to aid in surgical planning and for intraoperative guidance $[11,12]$. While this has proven to be a beneficial addition to the surgical environment, several caveats should be addressed. Each series of brain slices contained within the Schaltenbrand Wahren atlas were created from separate cadaver brain hemispheres. Digitizing and concatenating a slice series into a $3 \mathrm{D}$ volume will create an atlas representative of a single individual brain and not the general population. Varying interslice distances contained in the Schaltenbrand atlas results in the digitized atlas displaying jagged edges when viewed in planes orthogonal to the original slice. A smoothing operation is usually performed on the $3 \mathrm{D}$ volume. Due to intrinsic limitations within the anatomical atlases themselves, nonlinear registration and superposition over patient MRI provide only a close approximation of target loci.

\subsection{Goals and Clinical Motivation}

It is impossible to explore and map the functional organization of large portions of midbrain during a single stereotactic procedure. Surgeons must mentally reconstruct somatotopic organization sometimes based on as few as one 
exploratory trajectory alone within a single plane. In order to fully appreciate the functional organization within deep brain structures, complete functional maps would have to be created from whole individual brains. As this is not feasible, we must compromise by constructing a map of midbrain function based on multiple brains. It was our goal to combine intraoperatively derived functional data from a large population of movement disorder patients for compilation into a database of function with the following attributes. It must

- be capable of nonlinearly warping to match any patient MRI volume compensating for anatomical variability in three-dimensions;

- be fully searchable via an interactive database search engine;

- contain detailed somatotopic information relevant to Parkinson's Disease and movement disorder neurosurgery;

- possess compatibility with the image guided visualization platform currently under development at the RRI;

- allow integration of new patient data and new anatomical structures into the database with minimal user intervention, and

- present data in a manner interpreted easily by any user.

A functional database satisfying these criteria has the potential to facilitate preoperative planning of electrode trajectories and act as a somatotopic reference during the surgical procedure.

\section{Method}

\subsection{Data Collection}

All surgical procedures included in this study consisted of patients receiving left or right pallidotomies or thalamotomies involving mechanical lesion, leukotome (MNI) [11], RF lesioning (LHSC) or deep brain stimulator insertion (MNI, LHSC). Patients ranged from 25 to 75 years of age and included both Parkinson's Disease patients and essential tremor patients. In order to create a database of function representative of a specific population, certain patient exclusion criteria were necessary. Patients in the study did not have space occupying brain masses prior to or at time of surgery or other pathologies which might distort somatotopy. The inherent plasticity of the brain allows reorganization when afferent information is suddenly removed. Any patients with a physical condition that might alter the functional organization of their brain, such as an amputation or congenital anomaly, were also excluded. Patient verbal response provides a large proportion of data included in this atlas. Those patients deemed not fully coherent or totally aware of the procedure they were experiencing were excluded. Patients undergoing treatment for tremor resulting from other pathological origin such as trauma induced tremor, or multiple sclerosis were also excluded. 


\subsection{Standardizing Physiological Responses}

A protocol was developed to encode for all possible sensory or motor responses elicited at different stimulation voltages or locations. After designing a subdivided model based on Tasker's anatomical model for physiological data [4], anatomical numbers can be assigned to discreet areas of the body. To encode for sensory responses, we developed a list of response codes which could successfully describe every response produced by the 40 patients using stimulation and microcellular recording. All patients and each exploratory trajectory performed for that patient were assigned an identification number for inclusion into a tag code containing all pertinent information. All tag codes are of the format: patientID\#_trajectory\#_method_bodyside_bodypart\#_responsecode

For example, patient 12 felt a weak tingling sensation at the tip of his third digit, left hand during macrostimulation at $0.25 \mathrm{~V}$ on trajectory 1 . This response would be encoded as: 012_1_0.25V_L_66_PTw . This coding method was designed so an experienced user could tell at a glance, after searching the database, the patient origin of the data tag, which physiological method was used at what parameters, the side of the body eliciting the response, area of the body, and the evoked response. Tag codes of this nature make it possible to design highly flexible database search engines which search for any field or combination of fields within the tag code. In order to plot these codes onto the appropriate anatomical area of the patient MRI in three-dimensional space, images must be registered to the coordinate system defined by the stereotactic head frame. Registration may be performed manually by identifying the frame-based fiducial markers within 10 axial images then calculating a rigid transformation from MRI space to frame space or automatically using the frame finder aspect of our visualization platform.

When all codes for a particular patient are tagged to their respective MRI at appropriate coordinates, they are transformed into standard stereotactic space and added to the collective somatotopy. Interpatient similarities may then be analyzed.

\subsection{Nonlinear Warp Algorithm}

Nonlinear transformation matrices for each patient were calculated which accurately describe the warp from native MRI patient space to our high resolution reference brain in standard stereotactic space. This nonlinear transformation matrix was calculated for each patient using ANIMAL (Automatic Nonlinear Image Matching and Anatomical Labelling) [13], a nonlinear deformation algorithm that requires no user intervention or identification of homologous landmarks. Registration of the patient to the reference brain is accomplished by ANIMAL in two optimization steps: a linear component through automatic estimation of the best affine transformation matrix between the two volumes, and a nonlinear component, achieved through a hierarchial multi-scale, multi- resolution strategy. The end result is a nonlinear transformation matrix which maps the anatomy of the patient brain onto the anatomy of the reference brain. Each 
unique transformation matrix was used to resample the original patient data codes tagged onto their respective MRI s into reference brain stereotactic space. Inversion of a patient's nonlinear transformation and subsequent resampling of the functional atlas with this inverted matrix will transform all database codes into that patient's native MRI space.

\subsection{Searchable Database}

The usefulness of the database was maximized by permitting searches through every code for any combination of tag fields. A search engine was written using a scripting language, Python, that is compatible with the source code of the vtk/Python based image guided neurosurgical program under development at the RRI. We have incorporated an interactive query facility into this visualization platform to allow direct access to database entries. A digitized and segmented image of the anatomical model with point-and-click functionality linked to the database allow data codes pertinent to anatomy selected by the user to be extracted.

\section{Results}

We show here the results of some representative database searches displayed over the reference brain which provides information pertinent to PD surgery for surgical planning or intraoperative guidance. Since statistical significance of an atlas of this nature will increase with the number of patients added, most examples are taken from the left thalamotomy database currently containing the most patients. Functional data contained within the database is quite specific and encompasses a small area of midbrain. Prior to lesioning in a thalamotomy case, a neurosurgeon attempts to detect tremor cells located in the kinesthetic area of the thalamus using microelectrode recording. When tremor cells are detected within the suspected Vim region and the surrounding area sufficiently mapped, a lesion protocol is determined. Figure 1 shows a clustering of tremor cells in reference brain space that were detected in thalamotomy patients during microcellular recording. Once these data points are nonlinearly warped to a patient preoperative MRI, the surgeon may use these data as a predictor of tremor cell location and plan trajectories accordingly.

A functional border between kinesthetic cells detected via microcellular recording and sensory cells detected through microstimulation was observed (Figure 2, left). In agreement with the literature, kinesthetic cells lie anteriorly and slightly superior to the sensory neurons within the predicted Vc region. Note the delineation of a functional border where the two data types meet and slightly overlap. The asterisk indicates a trajectory which was determined intraoperatively as too posterior. Subsequent exploration within that patient was moved anteriorly based on the functional information provided by that trajectory.

We have gathered electrophysiological data bilaterally for the GPi. All neurons detected within the GPi were kinesthetic however a greater number of data 
codes have to be collected before displaying a clustered visualization. Outside of the GPi, clustering was noted around the optic tract located deep to the inferior GPi border (Figure 2, right). Responses surrounding the optic tract were from three patients who experienced visual phenomena in the form of flashing white lights during microstimulation.

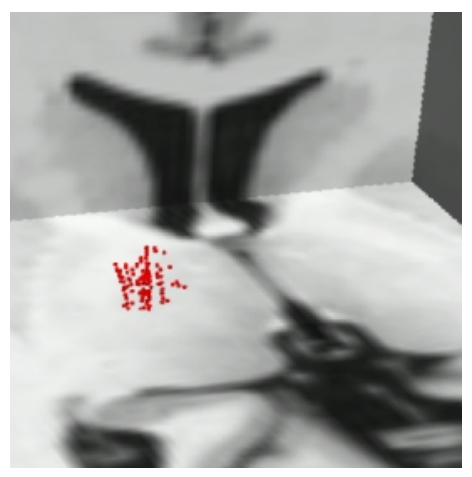

Fig. 1. Tremor cells (red spheres) located through microcellular recording during 12 different left thalamotomies.

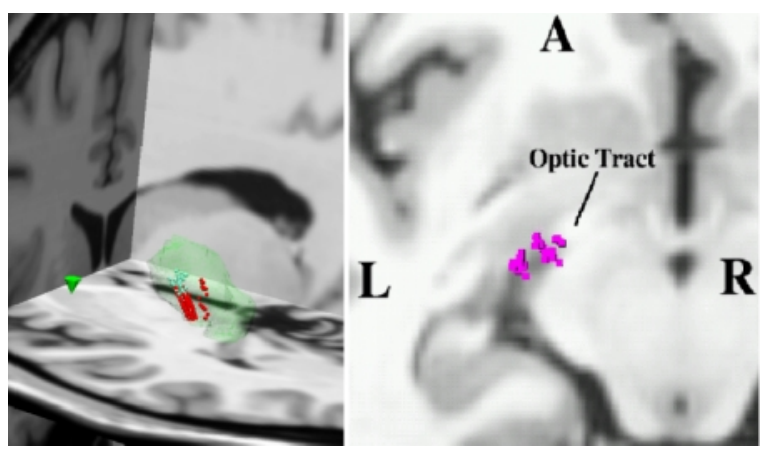

Fig. 2. Left:Functional border between Vim area (light blue spheres) and the Vc sensory area (red spheres). Surface rendered thalamus also shown in green. Right: Axial plane showing locations where microstimulation evoked visual phenomena during 7 different left pallidotomies. $\mathrm{L}=$ left, $\mathrm{R}=$ right, $\mathrm{A}=$ anterior

\section{Discussion}

Functional data superimposed within patient MRIs will have many applications. Preoperative planning will be facilitated through comparison of predicted lesion targets obtained through MRI localization with data provided by the database. Studies have shown that due to anatomical variability, the site of actual lesions 
can vary up to $5 \mathrm{~mm}$ from the original MRI based target site [1]. A functional atlas with nonlinear warping capabilities will accommodate the large variation of anatomical structures throughout a population and greatly assist target localization. Intraoperatively, patient responses evoked through electrophysiological exploration may be compared directly with the functional database as they occur. Direct consultation and comparison with the database will help the surgeon confirm location of the electrode tip within that patient's anatomy.

A probability database like this has the advantage that data points may easily be validated when added through simple comparison with existing points. All data, archived or current, are obtained through actual physiological mapping of human brain, and once coded require no further analysis prior to inclusion into the database. Following the addition of archived electrophysiological data from previous patients, a series of validations will be performed. Intraoperative validation will assess its predictive value during surgical guidance. Comparative studies will be possible for contrasting the functional organization displayed by the database with print atlases of function like Emmers and Tasker's physiological maps [14]. Data will be analyzed to elucidate the medial to lateral representation of the human body within thalamic nuclei at the finest resolution possible. Results of this analysis will be compared and contrasted with Hassler's map of thalamic homuncular organization [15]. Further elucidation of joint-based somatotopy observed within the pallidum will also be investigated. Plans for inclusion of other surgically relevant structures, the subthalamic nuclei for example, will be realized when data becomes available.

Ultimately, clustered population data representing one response type for a discrete area of the body will be displayed as a 3D fuzzy region of probability within the reference brain. This probability region will indicate an area of anatomy that, when explored electrophysiologically, would likely produce a specific response. The strength of prediction will be dependent on the amount of data the region represents and how tightly the population responses cluster around the centroid of the fuzzy region.

In addition, this database could prove useful for surgical training once fully integrated into RRI's surgical guidance program. Full three-dimensional appreciation of a very complex structure like a homunculus can be difficult to visualize from standard textbook images. Surgical residents would be provided with a unique and novel representation of three-dimensional somatotopic organization to increase their understanding of functional stereotactic procedures.

\section{Conclusions}

We have developed a robust and novel method to display intraoperatively derived functional data from a large population of patients within the space of one standard brain. Our method demonstrates delineation of functional borders within the thalamus and identifies high probability tremor areas. The resulting database contains all physiological responses recorded during 40 stereotactic procedures after standardization with comprehensive anatomical models and re- 
sponse codes. Nonlinearly warping data into native patient MRI space using the inverse nonlinear transform permits simultaneous display of physiological maps from several stereotactic procedures on different patients within the space of a patient's MRI data-set. Displaying physiological responses in this fashion allows real time comparison of the functional atlas with responses obtained during intraoperative stimulation and can facilitate preoperative planning.

\section{References}

1. Iacono R.P., Henderson J.M., Lonser R.R.: Combined stereotactic thalamotomy and posteroventral pallidotomy for Parkinson's Disease. Journal of Image Guided Neurosurgery 1:133-140, 1995 759, 766

2. Speelman J.D., Bosch D.A.: Resurgence of functional neurosurgery for Parkinson's Disease: A historical perspective. Movement Disorders 13(3):582-588, 1998759

3. Narabayashi H.: Pallidotomy revisited. Analysis of posteroventral pallidotomy. Stereotactic and Functional Neurosurgery 69(1-4 Pt.2):54-61, 1997759

4. Tasker R.R., Organ L.W., Hawrylyshyn P.A.: The Thalamus and Midbrain of Man, C.C. Thomas Publisher, Springfield, Illinois, USA. 1982 759, 760, 763

5. Keller T.M., Tcheng T.K., Burkhard P.R. Richard H., Tamas L.B.: Stereotactically guided thalamotomy for treatment of parkinsonian tremor isolated to the lower extremity. Journal of Neurosurgery 89:314-316, 1998759

6. Honda S., Seike M., Nishimura H., Kurisaka M., Mori K.: The distribution fields of sensory neurons in the human thalamus. Stereotactic and Functional Neurosurgery 67:218-230, 1996-97 759, 760

7. Lozano A., Hutchinson W., Kiss Z., Tasker R., Davis K., Dostrovsky J.: Methods for microelectrode-guided posteroventral pallidotomy. Journal ofNeurosurgery 84(2):194-202, 1996760

8. Guiot G., Hardy J., Albe-Fessard D.: Delimitation precise des structures sous-corticales et identification des noyaux thalamiques chez l'homme par l'electorphysiologie stereotaxique. Neurochirugia 5:1-18, 1962760

9. Benabid A.L., Benazzouz A., Hoffmann D., Limousin P., Krack P., Pollak P.: Longterm electrical inhibition of deep brain targets in movement disorders. Movement Disorders 13(Suppl3):119-125, 1998761

10. Schaltenbrand G., Wahren W.: Atlas for Stereotaxy of the Human Brain. Stuttgart: Georg Thieme Verlag. 1977761

11. St-Jean P., Sadikot A.F., Collins L., Clonda D., Kasrai R., Evans A.C.: Automated atlas integration and interactive three-dimensional visualization tools for planning and guidance in functional neurosurgery. IEEE Transactions on Medical Imaging 17(5):672-680, 1998 761, 762

12. Nowinski W.L., Fang A., Nguyen B.T., Raphel J.K., Jagannathan L., Raghavan R., Bryan R.N., Miller G.A.: Multiple brain atlas database and atlas-based neuroimaging system. Computer Aided Surgery 2:42-66, 1997761

13. Collins D.L., Holmes C.J., Peters T.M., Evans A.C.: Automatic 3-D model-based neuroanatomical segmentation. Human Brain Mapping 3:190-208, 1995763

14. Emmers R., Tasker R.R. The Human Somesthetic Thalamus. Raven Press, New York, 1975766

15. Hassler R.: Architectonic organization of the thalamic nuclei.: In G. Schaltenbrand, A.E. Walker(eds): Stereotaxy of the Human Brain. Stuttgart: Thieme, 1982766 